\title{
A Bijective Proof of a Major Index Theorem of Garsia and Gessel*
}

\author{
Mordechai Novick \\ Department of Mathematics \\ Hebrew University, Jerusalem, Israel \\ mordecha.novick@mail.huji.ac.il
}

Submitted: Jun 3, 2009; Accepted: Apr 7, 2010; Published: Apr 19, 2010

Mathematics Subject Classification: 05A05

\begin{abstract}
In this paper we provide a bijective proof of a theorem of Garsia and Gessel describing the generating function of the major index over the set of all permutations of $[n]=\{1, \ldots, n\}$ which are shuffles of given disjoint ordered sequences $\pi_{1}, \ldots, \pi_{k}$ whose union is $[n]$. The proof is based on a result (an "insertion lemma") of Haglund, Loehr, and Remmel which describes the change in major index resulting from the insertion of a given new element in any place in a given permutation. Using this lemma we prove the theorem by establishing a bijection between shuffles of ordered sequences and a certain set of partitions. A special case of Garsia and Gessel's theorem provides a proof of the equidistribution of major index and inversion number over inverse descent classes, a result first proved bijectively by Foata and Schutzenberger in 1978. We provide, based on the method of our first proof, another bijective proof of this result.
\end{abstract}

\section{Introduction}

In 1913, Percy MacMahon introduced the major index statistic, defined for any permutation $\sigma=\sigma_{1} \ldots \sigma_{n}$ of a multiset of integers of size $n$ as the sum of the descents of $\sigma$, i.e., $\operatorname{maj}(\sigma)=\sum_{i=1}^{n-1} i \chi\left(\sigma_{i}>\sigma_{i+1}\right)^{1}$. Let $[n]=\{1, \ldots, n\}$ and $[n]^{0}=\{0,1, \ldots, n\}$. Let $[n]_{q}=1+\cdots+q^{n-1},[n]_{q} !=\prod_{i=1}^{n}[i]_{q}$, and if $a_{1}, \ldots, a_{k}$ are positive integers which sum to $n$, then let

$$
\left[\begin{array}{c}
n \\
a_{1}, \ldots, a_{k}
\end{array}\right]_{q}=\frac{[n]_{q} !}{\left[a_{1}\right]_{q} ! \cdots\left[a_{k}\right]_{q} !} .
$$

*This paper is a condensed version of an M.Sc. thesis written under the direction of Gil Kalai of the Hebrew University and Yuval Roichman of Bar-Ilan University. I extend my thanks to both of them for their time and assistance.

${ }^{1}$ We adopt the convention that for any statement $A, \chi(A)=1$ if $A$ is true and $\chi(A)=0$ if $A$ is false. 
If $T$ denotes the multiset $\left\{1^{a_{1}}, \ldots k^{a_{k}}\right\}$ (i.e., the set of $a_{i}$ copies of the number $i$ for $i=$ $1, \ldots, k$, with $\left.\sum_{i=1}^{k} a_{i}=n\right)$, then MacMahon discovered that the generating function for the major index over the set $P(T)$ of permutations of $T$ is the following q-multinomial coefficient:

$$
\sum_{\sigma \in P(T)} q^{m a j(\sigma)}=\left[\begin{array}{c}
n \\
a_{1}, \ldots, a_{k}
\end{array}\right]_{q} .
$$

He went on to prove in [7] that this is also the generating function for the inversion number of permutations of the same set (an inversion of $\sigma$ is a pair $(i, j) \in[n-1] \times[n]$ with $i<j$ and $\sigma_{i}>\sigma_{j}$, and the inversion number is the total amount of such pairs: $\left.\operatorname{inv}(\sigma)=\sum_{i<j} \chi\left(\sigma_{i}>\sigma_{j}\right)\right)$. This proved that these two statistics are equidistributed over all the permutations of any multiset of integers. Specifically, in the case where $T=[n]$ (i.e., $a_{1}=\ldots=a_{n}=1$ ), and thus $P(T)=S_{n}$, we have:

$$
\sum_{\sigma \in S_{n}} q^{\operatorname{maj}(\sigma)}=\sum_{\sigma \in S_{n}} q^{i n v(\sigma)}=[n]_{q} !=(1+q) \cdots\left(1+q+q^{2}+\cdots+q^{n-1}\right) .
$$

In 1968, over fifty years after the work of MacMahon, Foata [2] gave a bijective proof of this equidistribution result. Ten years later, in 1978, Foata and Schutzenberger [3] showed that Foata's bijection preserves the inverse descent class of permutations so that the major index and the inversion number are also equidistributed over inverse descent classes of $S_{n}$. The following year, Garsia and Gessel [4] used Stanley's theory of P-partitions [9] to prove the following theorem. This theorem also immediately implies the equidistribution result of MacMahon as well as that of Foata and Schutzenberger.

Theorem ([4], Theorem 3.1): Let $\pi_{1}, \ldots, \pi_{k}$ be ordered complementary subsets of $[n]$, where $\pi_{i}$ has length $a_{i}$ for $i=1, \ldots, k$ (and hence $\left.a_{1}+\cdots+a_{k}=n\right)$. Let $\mathcal{S}\left(\pi_{1}, \ldots, \pi_{k}\right)$ be the collection of permutations of $[n]$ obtained by shuffling $\pi_{1}, \ldots, \pi_{k}$. Then

$$
\sum_{\sigma \in \mathcal{S}\left(\pi_{1}, \ldots, \pi_{k}\right)} q^{\operatorname{maj}(\sigma)}=\left[\begin{array}{c}
n \\
a_{1}, \ldots, a_{k}
\end{array}\right]_{q} q^{\operatorname{maj}\left(\pi_{1}\right)+\cdots+\operatorname{maj}\left(\pi_{k}\right)} .
$$

To see how MacMahon's results follow from this, define $a_{0}=0$, and let $\pi_{i}=\left(a_{0}+\cdots+\right.$ $\left.a_{i-1}, a_{0}+\cdots+a_{i-1}+1, \ldots, a_{0}+\cdots+a_{i}\right)$. Note that $\operatorname{maj}\left(\pi_{i}\right)=0$ for all $i$ in this case, so the " $q$ " term on the right is trivial. MacMahon's results then follow by noting that there is a simple bijective correspondence between $\mathcal{S}\left(\pi_{1}, \ldots, \pi_{k}\right)$ and the set of permutations of $\left\{1^{a_{1}}, \ldots k^{a_{k}}\right\}$ which preserves both inversion number and major index; just replace all elements of $\pi_{i}$ with the number $i$.

In this paper we provide a bijective proof of this theorem. We actually prove the following theorem, from which the above result follows:

Theorem 1.1: Let $\pi$ be an ordered subset of $[n]$ of length $a$, and let $\theta$ be an ordering of $[n]-\{\pi\}$. Let $\mathcal{S}(\theta, \pi)$ be the collection of permutations of $[n]$ obtained by shuffling $\theta$ and $\pi$. Then

$$
\sum_{\sigma \in \mathcal{S}(\theta, \pi)} q^{\operatorname{maj}(\sigma)}=\left[\begin{array}{l}
n \\
a
\end{array}\right]_{q} q^{\operatorname{maj}(\theta)+\operatorname{maj}(\pi)} .
$$


To obtain the version proved by Garsia and Gessel, simply apply Theorem 1.1 inductively on $i=2, \ldots, k$ with $\theta$ some shuffle of $\pi_{1}, \ldots, \pi_{i-1}$ and with $\pi=\pi_{i}$. Summing over all $\theta$ and applying the inductive assumption then yields:

$$
\begin{aligned}
\sum_{\sigma \in \mathcal{S}\left(\pi_{1}, \ldots, \pi_{i}\right)} q^{\operatorname{maj}(\sigma)}= & {\left[\begin{array}{c}
a_{1}+\cdots+a_{i} \\
a_{i}
\end{array}\right]_{q} \times } \\
& \left(\left[\begin{array}{c}
a_{1}+\cdots+a_{i-1} \\
a_{1}, \ldots, a_{i-1}
\end{array}\right]_{q} q^{\operatorname{maj}\left(\pi_{1}\right)+\cdots+m a j\left(\pi_{i-1}\right)}\right) q^{\operatorname{maj}\left(\pi_{i}\right)} \\
= & {\left[\begin{array}{c}
a_{1}+\cdots+a_{i} \\
a_{1}, \ldots, a_{i}
\end{array}\right]_{q} q^{\operatorname{maj}\left(\pi_{1}\right)+\cdots+m a j\left(\pi_{i}\right)} . }
\end{aligned}
$$

When $i=k$ this clearly becomes equation (4).

Our main tool in proving Theorem 1.1 will be a lemma of Haglund, Loehr, and Remmel. This lemma describes the increase in major index resulting from the insertion of a given element at any index in a given permutation, and it immediately implies MacMahon's result on the equidistribution of inversion number and major index (a special case of Theorem 1.1, as noted). After using this lemma to prove the theorem, we return to the topic of inverse descent classes and show how our work leads to a new bijective proof of that equidistribution result as well.

\section{Preliminaries}

This section introduces the terminology and notation that will be used in the remainder of the paper (aside from what has been defined in the introduction). An element $\sigma \in S_{n}$ is considered both as a word $\sigma_{1} \sigma_{2} \ldots \sigma_{n}$ (whose individual elements $\sigma_{1}, \sigma_{2}, \ldots$ we call letters), and as a bijection from $[n]$ to itself, with $\sigma(i)=\sigma_{i}$ for $i=1, \ldots, n$. A subword of $\sigma$ is a string of the form $\sigma_{i_{1}} \sigma_{i_{2}} \ldots \sigma_{i_{m}}$ for some $m \leqslant n$ such that $1 \leqslant i_{1}<\ldots<i_{m} \leqslant n$. The permutation $\sigma$ can be identified with the ordered sequence $\mathbf{a}=\left(\sigma_{1}, \sigma_{2}, \ldots, \sigma_{n}\right)$, and conversely any ordered sequence of distinct integers can be identified with a permutation in the obvious manner. The $k$-initial segment of a (for $k<n)$ is the subsequence $\left(\sigma_{1}, \ldots, \sigma_{k}\right)$. $\operatorname{set}(\mathbf{a})$ denotes the (unordered) set of elements contained in $\mathbf{a}$.

An index $i$ is a descent of $\sigma$ if $\sigma_{i}>\sigma_{i+1}$, and the descent set of $\sigma$ (denoted Des $(\sigma)$ ) is defined as $\operatorname{Des}(\sigma):=\left\{i \in[n-1]: \sigma_{i}>\sigma_{i+1}\right\}$. We denote by $d_{k}(\sigma)$ the number of descents in $\sigma$ greater than or equal to $k$ (i.e., the number of descents at or to the right of $\sigma_{k}$; specifically, $d_{1}(\sigma)=|\operatorname{Des}(\sigma)|$ ) (we also refer to this quantity as $\operatorname{des}(\sigma)$ ). Indices of $\sigma$ that are not descents are called ascents. It is easily observed that $\operatorname{maj}(\sigma)=\sum_{k=1}^{n} d_{k}(\sigma)$, as a descent at index $i$ is "accounted for" exactly $i$ times in the sum on the right.

We end this section by defining two new functions. Our approach will be based on a 
study of what happens to the major index of a permutation $\sigma$ of a set $T$ of $n$ distinct positive integers when some other integer $r$ is inserted in the $k$-th position (i.e., after $\sigma_{k}$, or at the left end if $\left.k=0\right)$ to create a new permutation which we denote $\sigma^{(r, k)}$. Define $m i_{(\sigma, r)}(k):=\operatorname{maj}\left(\sigma^{(r, k)}\right)-\operatorname{maj}(\sigma)$ (the initials stand for major increment). We will also be interested in the major increment sequence of $\sigma$ relative to $r$ defined as $M I S(\sigma, r):=\left(m i_{(\sigma, r)}(0), \ldots, m i_{(\sigma, r)}(n)\right)$. In words, the major increment sequence of $\sigma$ relative to $r$ is the sequence of $n+1$ numbers whose $i$-th entry $(0 \leqslant i \leqslant n)$ denotes the change in major index induced by inserting $r$ into $\sigma$ at the $i$-th position.

Example 2.1. Let $T=[6]$. Inserting $r=7$ into the permutation $\sigma=426351$, which has major index $9(=1+3+5)$.

\begin{tabular}{cccc}
$k$ & $\sigma^{(r, k)}$ & $\operatorname{maj}\left(\sigma^{(r, k)}\right)$ & $m i_{(\sigma, r)}(k)$ \\
\hline 0 & 7426351 & 13 & 4 \\
1 & 4726351 & 12 & 3 \\
2 & 4276351 & 14 & 5 \\
3 & 4267351 & 11 & 2 \\
4 & 4263751 & 15 & 6 \\
5 & 4263571 & 10 & 1 \\
6 & 4263517 & 9 & 0
\end{tabular}

Thus we have $M I S(\sigma, r)=(4,3,5,2,6,1,0)$. Note that this sequence is a permutation of $[6]^{0}$. This is no accident, as we will see in the next section.

\section{$3 \quad$ Proving Theorem 1.1 Using the Insertion Lemma}

When $a=1$, we have

$$
\left[\begin{array}{l}
n \\
a
\end{array}\right]_{q}=\left[\begin{array}{c}
n \\
1
\end{array}\right]_{q}=\frac{q^{n}-1}{q-1}=\left(1+q+\cdots+q^{n-1}\right)
$$

and thus Theorem 1.1 reads as follows: Given $n$ and $r \in[n+1]$, let $\theta$ be any permutation of $[n+1]-\{r\}$. Then

$$
\sum_{k=0}^{n} q^{\operatorname{maj}\left(\theta^{(r, k)}\right)}=\left(1+q+\cdots+q^{n}\right) q^{\operatorname{maj}(\theta)} .
$$

Dividing both sides by $q^{\operatorname{maj}(\theta)}$ yields:

$$
\sum_{k=0}^{n} q^{m a j\left(\theta^{(r, k)}\right)-m a j(\theta)}=\sum_{i=0}^{n} q^{m i_{(\theta, i)}(r)}=1+q+\cdots+q^{n} .
$$

In words: The sequence $M I S(\theta, r):=\left(m i_{(\theta, r)}(0), \ldots, m i_{(\theta, r)}(n)\right)$ is a permutation of $[n]^{0}$. This fact (phrased in very different terminology) was first noted by Gupta [5]. It was 
demonstrated also by a more general "insertion lemma" of Haglund, Loehr, and Remmel ([6], Lemma 4.1), whose result we now explain.

Let $T=\left\{t_{1}, \ldots, t_{n}\right\}$ denote some set of distinct integers and let $r$ be some integer not in $T$. We label the spaces in which $r$ can be inserted into a permutation $\sigma=\sigma_{1} \ldots \sigma_{n}$ of $T$ from left to right with the integers $0, \ldots, n$ (so that the space after $\sigma_{i}$ is labeled $i$ for $i=1, \ldots, n$ and the space preceding $\sigma_{1}$ is labeled 0$)$. In this ordered labeling we refer to the space labeled $k$ as the $k$-th space (note that this means, e.g., that the "4th space" is actually the fifth from the left, as the leftmost space is labeled 0). We now relabel these spaces with a canonical labeling which we define as follows. A space (originally) labeled $i$ is called an $R L$-space of $\sigma$ relative to $r$ if it meets one of the following conditions:

1. $i=n$ and $\sigma_{n}<r$

2. $i=0$ and $r<\sigma_{1}$

3. $0<i<n$ and $\sigma_{i}>\sigma_{i+1}>r$

4. $0<i<n$ and $r>\sigma_{i}>\sigma_{i+1}$

5. $0<i<n$ and $\sigma_{i}<r<\sigma_{i+1}$.

Any space not called an RL-space of $\sigma$ relative to $r$ is called an $L R$-space of $\sigma$ relative to $r$. Intuitively, an LR-space is a space where the insertion of $r$ creates a "new descent" in $\sigma$, thus increasing the major index by at least the index of insertion. An RL-space is one where no new descent is created, and thus any increase in major index resulting from the insertion of $r$ is due solely to the "bumping" of pre-existing descents one index higher.

To produce the canonical labeling of $\sigma$ relative to $r$, we label the RL-spaces from right to left (hence the name) with the new labels $0, \ldots, k-1$ (where $k$ is the total number of RL-spaces) and we label the LR-spaces from left to right with the new labels $k, \ldots, n$. As an example, if:

$$
r=5, T=[10]-\{5\}, \sigma=1019827436
$$

then the RL-spaces of $\sigma$ relative to $r$ are $0,2,3,5,7$, and 8. Thus the canonical labeling of $\sigma$ looks as follows:

$$
{ }_{5} 10_{\overline{6}} 1_{\overline{4}} 9_{\overline{3}} 8_{\overline{7}} 2 \overline{2}_{2} 7_{\overline{8}} 4_{\overline{1}} 3 \overline{0} 6 \overline{9} .
$$

Let us denote the label of the $k$-th space in the canonical labeling of $\sigma$ relative to $r$ as $l a b_{(\sigma, r)}(k)$. Then the result of Haglund, Loehr, and Remmel can be expressed as follows:

Lemma 3.1- Insertion Lemma ([6], Lemma 4.1):

$$
\operatorname{maj}\left(\sigma^{r, k}\right)=\operatorname{maj}(\sigma)+l a b_{(\sigma, r)}(k) .
$$

In other words,

$$
l a b_{(\sigma, r)}(k)=m i_{(\sigma, r)}(k)
$$

so that $M I S(\sigma, r)$ is just the canonical labeling, read off from left to right. Specifically, in the case that $T=[n+1]-\{r\}$ for some $n$ and $r$, this lemma states that $M I S(\sigma, r)$ is 
a permutation of $[n]^{0}$, proving Theorem 1.1 in the case of $a=1$.

We now proceed to the general case (i.e., $a>1$ ) of Theorem 1.1. Let $b=n-a$ denote the length of $\theta$. We will establish a bijection $\Phi$ between the set $\mathcal{S}(\theta, \pi)$ of shuffles of $\theta$ and $\pi$ and the set $\mathcal{P}(b, a)$ of partitions containing $a$ parts (some of which may be zero) all less than or equal to $b$. Both of these sets have cardinality $\left(\begin{array}{c}a+b \\ a\end{array}\right)=\left(\begin{array}{l}n \\ a\end{array}\right)$. In the case of $\mathcal{S}(\theta, \pi)$, a shuffle is determined by the $a$-element subset of $[n]$ corresponding to the positions where the elements of $\pi$ are placed, and in the case of $\mathcal{P}(b, a)$, a partition $\lambda=\left(\lambda_{1}, \ldots, \lambda_{a}\right)$ with $0 \leqslant \lambda_{1} \leqslant \ldots \leqslant \lambda_{a} \leqslant b$ corresponds to the $a$-element subset $\left\{\lambda_{i}+i: i=1, \ldots, a\right\}$ of $[n]$. Given $\lambda=\left(\lambda_{1}, \ldots, \lambda_{a}\right) \in \mathcal{P}(b, a)$, denote the sum $\sum_{i=1}^{a} \lambda_{i}$ as $|\lambda|$. Our bijection $\Phi: \mathcal{S}(\theta, \pi) \rightarrow \mathcal{P}(b, a)$ will have the property that for $\sigma \in \mathcal{S}(\theta, \pi)$,

$$
\operatorname{maj}(\sigma)=\operatorname{maj}(\theta)+\operatorname{maj}(\pi)+|\Phi(\sigma)| .
$$

Raising $q$ to both sides of this equation, summing the left side over $\mathcal{S}(\theta, \pi)$ and the right side over $\mathcal{P}(b, a)$ (which preserves equality, by the bijection), yields:

$$
\sum_{\sigma \in \mathcal{S}(\theta, \pi)} q^{\operatorname{maj}(\sigma)}=q^{\operatorname{maj}(\theta)+m a j(\pi)} \sum_{\lambda \in \mathcal{P}(b, a)} q^{|\lambda|}
$$

As is well-known, the generating function for the sums of the partitions in $\mathcal{P}(b, a)$ can be expressed as a $q$-binomial coefficient:

$$
\sum_{\lambda \in \mathcal{P}(b, a)} q^{|\lambda|}=\left[\begin{array}{c}
b+a \\
a
\end{array}\right]_{q}=\left[\begin{array}{l}
n \\
a
\end{array}\right]_{q} .
$$

In fact, some sources define the $q$-binomial coefficient in this manner; see, e.g., ([1], chapter 3). Thus, this bijection proves Theorem 1.1.

To define our bijection we need some new notation. Given $\sigma \in \mathcal{S}(\theta, \pi)$, we imagine that $\sigma$ is constructed by the insertion of $\pi$ into $\theta$ one letter at a time, the letters being inserted in the reverse of their order of appearance in $\pi$ (i.e., if $\pi=(\pi(1), \ldots, \pi(a))$, then $\pi(a)$ is inserted first and $\pi(1)$ is inserted last). Note that every insertion occurs to the left of the previous one. Let $\sigma^{i}$ denote the subword of $\sigma$ consisting of $\theta$ and the elements $\pi(i), \ldots, \pi(a)$, so that $\sigma^{a}, \sigma^{a-1}, \ldots, \sigma^{1}=\sigma$ represent the intermediate steps of the insertion procedure just described. By convention, we define $\sigma^{a+1}=\theta$. Let $k_{i}$ denote the position at which $\pi(i)$ is inserted into $\sigma^{i+1}$ to yield $\sigma^{i}$. Since every insertion occurs to the left of the previous one, we have $k_{1} \leqslant \ldots \leqslant k_{a}$.

With this construction procedure, let $m_{i}=\operatorname{maj}\left(\sigma^{i}\right)-\operatorname{maj}\left(\sigma^{i+1}\right)$ (i.e., $m_{i}$ denotes the increase in major index induced by the insertion of $\pi(i))$ and let $t_{i}=m_{i}-d_{i}(\pi)$. We claim the following:

Theorem 3.2: The mapping $\Phi: \mathcal{S}(\theta, \pi) \rightarrow \mathcal{P}(b, a)$ defined by $\Phi(\sigma)=\operatorname{set}\left(\left(t_{1}, \ldots, t_{a}\right)\right)$ is a bijection between $\mathcal{S}(\theta, \pi)$ and $\mathcal{P}(b, a)$.

Example 3.3. Let $\theta=5274, \pi=631$, and $\sigma=5276341$. Then $\operatorname{maj}(\theta)=4$, and we have: 


$$
\begin{array}{llll}
\sigma^{3}=52741 & k_{3}=4 & \operatorname{maj}\left(\sigma^{3}\right)=8 & m_{3}=4 \\
\sigma^{2}=527341 & k_{2}=3 & \operatorname{maj}\left(\sigma^{2}\right)=9 & m_{2}=1 \\
\sigma^{1}=5276341 & k_{1}=3 & \operatorname{maj}\left(\sigma^{1}\right)=14 & m_{1}=5 .
\end{array}
$$

Thus in this example, $t_{1}=5-2=3, t_{2}=1-1=0$, and $t_{3}=4-0=4$, so $\Phi(\sigma)=$ $\{0,3,4\} \in \mathcal{P}(b, a)$.

$\Phi$ indeed satisfies property (11):

$$
\operatorname{maj}(\sigma)-\operatorname{maj}(\theta)=\sum_{i=1}^{a} m_{i}=\sum_{i=1}^{a} d_{i}(\pi)+\sum_{i=1}^{a}\left(m_{i}-d_{i}(\pi)\right)=\operatorname{maj}(\pi)+|\Phi(\sigma)| .
$$

It remains to show that $\Phi$ is indeed a bijection, and the remainder of this section is devoted to proving this fact. It is not immediately clear that $\Phi$ even maps $\mathcal{S}(\theta, \pi)$ into $\mathcal{P}(b, a)$ at all. To prove both that $\Phi(\sigma) \in \mathcal{P}(b, a)$ for all $\sigma \in \mathcal{S}(\theta, \pi)$ and that $\Phi$ is a bijection we need to have some idea of what the sequences $\operatorname{MIS}\left(\sigma^{i+1}, \pi_{i}\right)$ look like (for $i=1, \ldots, a)$. To this end we use the Insertion Lemma to prove the following:

Lemma 3.4: Let $\tau$ be a permutation of length $n$ and $p, q \notin \tau$. Then for any $j \leqslant n$, the first $j$ elements of $M I S\left(\tau^{(p, j-1)}, q\right)$ are some permutation of the set $\{x+\chi(q>p) \mid x$ is in the $j$-initial segment of $M I S(\tau, p)\}$.

Example 3.5. Let $\tau=436152, p=8, q=7$, and $j=5$. Then $\tau^{(p, j-1)}=4361852$, and $\chi(q>p)=0$. A quick calculation yields $M I S(\tau, 8)=(4,3,5,2,6,1,0)$ and $\operatorname{MIS}\left(\tau^{(p, j-1)}, 7\right)=(5,4,6,3,2,7,1,0)$. The first five elements of these two sequences are indeed the same. Reversing the values of $p$ and $q$, we have $\tau^{(p, j-1)}=$ 4361752, $\chi(q>p)=1, M I S(\tau, 7)=(4,3,5,2,6,1,0)$ and $M I S\left(\tau^{(p, j-1)}, 8\right)=$ $(5,4,6,3,7,2,1,0)$. The first five elements of the first sequence, each increased by 1 , yield the first five elements of the second sequence. Coincidentally, the order is preserved in this case, but this need not be true in general.

Proof (of lemma): By the Insertion Lemma, the first $j$ elements of $M I S(\tau, p)$ are the labels of the first $j$ spaces in the canonical labeling of $\tau$ relative to $p$, and similarly for $\operatorname{MIS}\left(\tau^{(p, j-1)}, q\right)$. Note that in any canonical labeling of a permutation $\sigma$, the labels of the first $j$ spaces are determined by the label of the last RL-space (resp., LR-space) which appears before $\sigma_{j}$; specifically, if this label is $k$ (resp., $k+j-1$ ), then the labels of the first $j$ spaces are $\{k, k+1, \ldots k+j-1\}$, in some order. So in the case of $p>q$ (Case A) we need only show that the label of the last RL-space (resp., LR-space) among the first $j$ spaces in $\tau^{(p, j-1)}$ (relative to $q$ ) is equal to that of the last RL-space (resp., LR-space) among the first $j$ spaces in $\tau$ (relative to $p$ ); and in the case of $q>p$ (Case B), we need to show that the former label is the successor of the latter. For ease of notation, in the following cases let $d$ denote the quantity $\operatorname{des}\left(\tau_{j} \ldots \tau_{n}\right)$.

Case A1: $p>q>\tau_{j-1}>\tau_{j}$ : In this case, the $(j-1)$-th space of $\tau$ is an RL-space relative

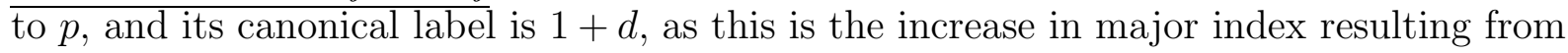
the insertion of $p$ between $\tau_{j-1}$ and $\tau_{j}$. The $(j-1)$-th space of $\tau^{(p, j-1)}$ is also an RL-space 
relative to $q$, and its canonical label is $\operatorname{des}\left(p \tau_{j} \ldots \tau_{n}\right)=1+d$, since $p>\tau_{j}$. Thus both $(j-1)$-spaces are RL-spaces and they have the same label, proving the result.

Case A2: $p>\tau_{j-1}>\max \left(q, \tau_{j}\right)$ : The $(j-1)$-th space of $\tau$ is as in Case A1, with label $1+d$. Since the canonical label of the last RL-space among the first $j$ spaces is $1+d$, the label of the last LR-space among these spaces must be $j+d$. The $(j-1)$-th space of $\tau^{(p, j-1)}$ is an LR-space relative to $q$, and its canonical label is $(j-1)+\operatorname{des}\left(p \tau_{j} \ldots \tau_{n}\right)=(j-1)+(1+d)$ (since $\left.p>\tau_{j}\right)=j+d$, proving the result.

Case A3: $\tau_{j-1}>p>\max \left(q, \tau_{j}\right)$ or $p>\max \left(q, \tau_{j}\right)>\min \left(q, \tau_{j}\right)>\tau_{j-1}: \quad$ In these cases, the $(j-1)$-th space of $\tau$ is an LR-space relative to $p$, and its canonical label is $j+d$. Thus the canonical label of the last RL-space among the first $j$ spaces must be $1+d$. The $(j-1)$-th space of $\tau^{(p, j-1)}$ is an RL-space relative to $q$, and its canonical label is $\operatorname{des}\left(p \tau_{j} \ldots \tau_{n}\right)=1+d\left(\right.$ since $\left.p>\tau_{j}\right)$, proving the result.

Case A4: $\left.p>\tau_{j}>\tau_{j-1}>q\right)$ : The $(j-1)$-th space of $\tau$ is as in Case A3, with label $j+d$. The $(j-1)$-th space of $\tau^{(p, j-1)}$ is also an LR-space relative to $q$, and its canonical label is $(j-1)+\operatorname{des}\left(p \tau_{j} \ldots \tau_{n}\right)=(j-1)+(1+d)$ (since $\left.p>\tau_{j}\right)=j+d$, proving the result.

Case A5: $\tau_{j-1}>\tau_{j}>p>q$ or $\tau_{j}>p>q>\tau_{j-1}$ : In this case, the $(j-1)$-th space of $\tau$ is an RL-space relative to $p$, and its canonical label is $d$. The $(j-1)$-th space of $\tau^{(p, j-1)}$ is also an RL-space relative to $q$, and its canonical label is $\operatorname{des}\left(p \tau_{j} \ldots \tau_{n}\right)=d$ (since $\left.p<\tau_{j}\right)$, proving the result.

Case A6: $\tau_{j}>p>\tau_{j-1}>q$ : The $(j-1)$-th space of $\tau$ is as in Case A5, with label $d$. Thus the canonical label of the last LR-space among the first $j$ spaces must be $(j-1)+d$. The $(j-1)$-th space of $\tau^{(p, j-1)}$ is an LR-space relative to $q$, and its canonical label is $\left.(j-1)+\operatorname{des}\left(p \tau_{j} \ldots \tau_{n}\right)=(j-1)+d\right)\left(\right.$ since $\left.p<\tau_{j}\right)$, proving the result.

Case A7: $\tau_{j}>\tau_{j-1}>p>q$ : In this case, the $(j-1)$-th space of $\tau$ is an LR-space relative to $p$, and its canonical label is $(j-1)+d$. Thus the canonical label of the last RL-space among the first $j$ spaces must be $d$. The $(j-1)$-th space of $\tau^{(p, j-1)}$ is an RL-space relative to $q$, and its canonical label is $\operatorname{des}\left(p \tau_{j} \ldots \tau_{n}\right)=d$ ) (since $p<\tau_{j}$ ), proving the result.

Case B1: $q>p>\tau_{j-1}>\tau_{j}$ : The $(j-1)$-th space of $\tau$ is as in Case A1, with label $1+d$. Thus the canonical label of the last LR-space among the first $j$ spaces must be $j+d$. The $j$ th space of $\tau^{(p, j-1)}$ is an LR-space relative to $q$, and its canonical label is $j+\operatorname{des}\left(p \tau_{j} \ldots \tau_{n}\right)=$ $j+(1+d)$ (since $\left.p>\tau_{j}\right)$, proving the result.

Case B2: $q>\tau_{j-1}>p>\tau_{j}$ : The $(j-1)$-th space of $\tau$ is as in Case A3, with label $j+d$. Thus the canonical label of the last RL-space among the first $j$ spaces must be $1+d$. The $(j-1)$-th space of $\tau^{(p, j-1)}$ is an RL-space relative to $q$, and its canonical label is $1+\operatorname{des}\left(p \tau_{j} \ldots \tau_{n}\right)=2+d\left(\right.$ since $\left.p>\tau_{j}\right)$, proving the result.

Case B3: $q>\tau_{j-1}>\tau_{j}>p$ : The $(j-1)$-th space of $\tau$ is as in Case A5, with label $d$. The $(j-1)$-th space of $\left.\tau^{(p, j}-1\right)$ is also an RL-space relative to $q$, and its canonical label is $1+\operatorname{des}\left(p \tau_{j} \ldots \tau_{n}\right)=1+d\left(\right.$ since $\left.p<\tau_{j}\right)$, proving the result.

Case B4: $\tau_{j-1}>q>p>\tau_{j}$ or $q>p>\tau_{j}>\tau_{j-1}$ : The $(j-1)$-th space of $\tau$ is as in Case 
A3, with label $j+d$. The $(j-1)$-th space of $\tau^{(p, j-1)}$ is also an LR-space relative to $q$, and its canonical label is $j+\operatorname{des}\left(p \tau_{j} \ldots \tau_{n}\right)=(j+1)+d$ (since $\left.p>\tau_{j}\right)$, proving the result.

Case B5: $\tau_{j-1}>\max \left(\tau_{j}, q\right)>\min \left(\tau_{j}, q\right)>p$ or $\min \left(q, \tau_{j}\right)>p>\tau_{j-1}$ : The $(j-1)$-th space of $\tau$ is as in Case A5, with label $d$. Thus the canonical label of the last LR-space among the first $j$ spaces must be $(j-1)+d$. The $(j-1)$-th space of $\tau^{(p, j-1)}$ is an LR-space relative to $q$, and its canonical label is $j+\operatorname{des}\left(p \tau_{j} \ldots \tau_{n}\right)=j+d$ (since $p<\tau_{j}$ ), proving the result.

Case B6: $\min \left(q, \tau_{j}\right)>\tau_{j-1}>p$ : The $(j-1)$-th space of $\tau$ is as in Case A7, with label

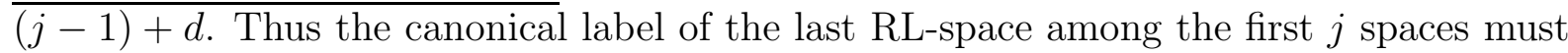
be $d$. The $(j-1)$-th space of $\tau^{(p, j-1)}$ is an RL-space relative to $q$, and its canonical label is $1+\operatorname{des}\left(p \tau_{j} \ldots \tau_{n}\right)=1+d$ (since $\left.p<\tau_{j}\right)$, proving the result.

Case B7: $\tau_{j}>\tau_{j-1}>q>p$ : The $(j-1)$-th space of $\tau$ is as in Case A7, with label $(j-$

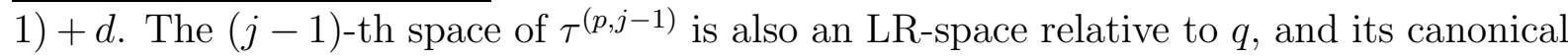
label is $j+\operatorname{des}\left(p \tau_{j} \ldots \tau_{n}\right)=j+d$ (since $p<\tau_{j}$ ), proving the result.

Q.E.D.

To apply the lemma to our case, let $\tau=\sigma^{i+1}, p=\pi(i)$, and let $j=k_{i}$ (so that $\tau^{(p, j)}=\sigma^{i}$ ). Finally, let $q=\pi(i-1)$. Note that $m_{i}=\operatorname{MIS}\left(\sigma^{i+1}, \pi(i)\right)\left(k_{i}\right)$, and that $m_{i-1}$ must be one of $\operatorname{MIS}\left(\sigma^{i}, \pi(i-1)\right)(0), \ldots, M I S\left(\sigma^{i}, \pi(i-1)\right)\left(k_{i}\right)$ because $\pi(i-1)$ must be inserted to the left of $\pi(i)$. We thus conclude, by Lemma 4.1, the following: If $\pi(i-1)<\pi(i)$, then the only possible values of $m_{i-1}$ lie to the left of $m_{i}$ in $M I S\left(\sigma^{i+1}, \pi(i)\right)$, including $m_{i}$ itself; and if $\pi(i-1)>\pi(i)$, then the only possible values of $m_{i-1}$ are the values to the left of $m_{i}$ in $M I S\left(\sigma^{i+1}, \pi(i)\right)$ (again, including $m_{i}$ itself), each incremented by 1 .

Example 3.6. Let $\sigma^{4}=\theta=6152, \pi=437$, and $\sigma^{1}=\sigma=6143572$ (so that $\sigma^{3}=61572$ and $\left.\sigma^{2}=613572\right)$. Then $k_{3}=3$ and $\operatorname{MIS}\left(\sigma^{4}, 7\right)=(3,2,4,1,0)$ (the italicized element being $\left.m_{3}\right)$. By the lemma, we expect the first four elements of $\operatorname{MIS}\left(\sigma^{3}, 3\right)$ the sole candidates for $m_{2}$ - to be some permutation of $(3,2,4,1)$ (because $3<$ $7)$. Indeed, the 4 -initial segment of $\operatorname{MIS}\left(\sigma^{3}, 3\right)$ is $(2,3,1,4)$ (the italicized element denoting $m_{2}$, as 3 is inserted at index $k_{2}=2$ to yield $\sigma_{2}$ ). Again by the lemma, we expect the first three elements of $M I S\left(\sigma^{2}, 4\right)$ - the candidates for $m_{1}$ - to be some permutation of $(3,4,2)$ (each of the first three elements of $\operatorname{MIS}\left(\sigma^{3}, 3\right)$ increased by 1 because $4>3)$ and indeed the 3 -initial segment of $M I S\left(\sigma^{2}, 4\right)$ is $(3,4,2)$ itself.

We can now easily prove the following proposition:

Proposition 3.7: Let $S_{i} \subseteq[n]$ be the set of elements contained in the $k_{i}$-initial segment of $M I S\left(\sigma^{i+1}, \pi(i)\right)$ (for $\left.i=1, \ldots, a\right)$ and let $T_{i}=S_{i}-d_{i}(\pi)=\left\{s-d_{i}(\pi) \mid s \in S_{i}\right\}$. Then $T_{1} \subseteq \ldots T_{a} \subseteq[b]$.

Proof (of proposition): By induction on the subscript of $T$, moving backwards from $a$ to 1 . For $i=a$ this is simply an application of the Insertion Lemma, as we have seen. Suppose the proposition is true for $i=m+1, \ldots, a$. If $\pi(m)<\pi(m+1)$ then $d_{m}(\pi)=$ $d_{m+1}(\pi)$ and $T_{m} \subseteq T_{m+1}$ iff $S_{m} \subseteq S_{m+1}$; if $\pi(m)>\pi(m+1)$ then $d_{m}(\pi)=d_{m+1}(\pi)+1$, and $T_{m} \subseteq T_{m+1}$ iff $S_{m} \subseteq\left\{s+1 \mid s \in S_{m+1}\right\}$. Both statements about the sets $S_{m}$ and $S_{m+1}$ 
are true by the lemma, as explained following the lemma and as illustrated in Example 4.3 .

Q.E.D.

Noting that $m_{i} \in S_{i}$ (as by definition, $m_{i}=\operatorname{MIS}\left(\sigma^{i+1}, \pi(i)\right)\left(k_{i}\right)$ ), we immediately have:

Corollary: For all $i \in[a], t_{i}=m_{i}-d_{i}(\pi) \in[b]$.

By this corollary, $0 \leqslant t_{i} \leqslant b$ for all $i$, and thus $\operatorname{set}\left(\left(t_{1}, \ldots, t_{a}\right)\right)$ is a partition in $\mathcal{P}(b, a)$. Hence $\Phi$ maps $\mathcal{S}(\theta, \pi)$ into $\mathcal{P}(b, a)$, as claimed.

It remains only to show that $\Phi$ is injective and surjective. We do this by explaining how to find the unique $\sigma=\Phi^{-1}(\lambda)$ for any given partition $\lambda=\left(\lambda_{1}, \ldots, \lambda_{a}\right) \in \mathcal{P}(b, a)$ ("unique", hence $\Phi$ is injective; "any", hence it is surjective). The elements of $\lambda$ comprise one representative each from the sets $T_{1}, \ldots, T_{a}$ defined in Proposition 4.1. By that proposition, these sets form a nested chain. For $i=a, \ldots, 1$, the choice of $m_{i}$ (and hence of $k_{i}$ ) determines both $t_{i}$ and the set $T_{i}$; specifically, the set $T_{i}$ contains precisely the first $k_{i}+1$ elements of $M I S\left(\sigma^{i+1}, \pi(i)\right)$ with $d_{i}(\pi)$ subtracted from each. Thus the only way to ensure that $T_{1} \subseteq \ldots \subseteq T_{a}$ is to choose $m_{i}$ to be the rightmost element of $\left\{\lambda_{i}+d_{i}(\pi) \mid i=1, \ldots, a\right\}$ which has not already been used in an earlier step, and thus $\Phi^{-1}(\lambda)$ is determined uniquely. This completes the proof of Theorem 3.2.

Q.E.D.

We illustrate the method of determining $\Phi^{-1}(\lambda)$ using Example 4.1., now being performed in reverse.

Example 3.8. We compute the permutation $\sigma=\Phi^{-1}(\{0,3,4\})$ (where $\theta=5274$ and $\pi=631$ as in Example 3.3). The values 0,3, and 4 are the differences $m_{i}-d_{i}(\pi)$ for $i=1,2,3$. For $i=3, d_{3}(\pi)=0$, and so $m_{3}$ must be 0,3 , or 4 . As $M I S(\theta, 1)=$ $(2,1,3,0,4)$, we must have $m_{3}=t_{3}=4$ (and hence $k_{3}=4, \sigma^{3}=52741$, and $\left.T_{3}=\{0,1,2,3,4\}\right)$ so that the remaining elements of $\lambda(0$ and 3$)$ are elements of $T_{3}$.

For the next step (the $i=2$ step) we look at these remaining elements, each incremented by $d_{2}(\pi)=1$ to yield 1 and 4 , and the $k_{3}$-initial segment of $\operatorname{MIS}\left(\sigma^{3}, 3\right)=$ $(3,4,2,1,5,0)$. The rightmost element in that segment among 1 and 4 is 1 , at position 3 , hence $m_{2}=1$ (and $\left.t_{2}=0\right), k_{2}=3, \sigma^{2}=527341$, and $T_{2}=\{0,1,2,3\}$.

Finally, the remaining element of $\lambda$ is 3 , now incremented by $d_{1}(\pi)=2$ to yield $m_{1}=5$. The $k_{2}$-initial segment of $\operatorname{MIS}\left(\sigma^{2}, 6\right)=(4,3,2,5,6,1,0)$ indeed contains this value at the fourth position, and thus $k_{1}=4$ and $\sigma^{1}=\sigma=5276341$, as desired.

\section{Equidistribution over Inverse Descent Classes}

The inverse descent class corresponding to a set $Q \subseteq[n]$ is the subset $S_{Q} \in S_{n}$ of all permutations of $[n]$ whose inverses (in the usual group-theoretic sense) have descent set $Q$. There is a simple and well-known combinatorial description of inverse descent classes: $k \in[n]$ is a descent of $\sigma^{-1}$ iff $k+1$ appears to the left of $k$ in $\sigma$. Thus, if $Q=\left\{q_{1}, \ldots, q_{t}\right\}$, then $S_{Q}$ is the set of shuffles of the complementary subsequences $\mathbf{q}_{\mathbf{0}}=\left(1, \ldots, q_{1}\right), \mathbf{q}_{\mathbf{1}}=$ 
$\left(q_{1}+1, \ldots, q_{2}\right), \ldots, \mathbf{q}_{\mathbf{t}}=\left(q_{t}+1, \ldots, n\right)$ such that none of these subsequences appears entirely to the right of any earlier subsequence. We refer to a shuffle with this latter property as a well-mixed shuffle. It is generally easier to deal with all shuffles of $\mathbf{q}_{\mathbf{0}}, \ldots, \mathbf{q}_{\mathbf{t}}$ rather than only the well-mixed ones. Thus we focus not on the set $S_{Q}$ itself but rather on the larger set of permutations with inverses whose descent set is any subset of $Q$. By the combinatorial description above, this is precisely the set of all shuffles of $\mathbf{q}_{\mathbf{0}}, \ldots, \mathbf{q}_{\mathbf{t}}$.

Applying the theorem of Gessel and Garsia to this set yields an especially neat result because each subsequence $\mathbf{q}_{\mathbf{i}}(i=0, \ldots, t)$ is increasing, hence $\operatorname{maj}\left(\mathbf{q}_{\mathbf{i}}\right)=0$. Thus we have:

$$
\sum_{\left\{\sigma \in S_{n} \mid \operatorname{Des}\left(\sigma^{-1}\right) \subseteq Q\right\}} q^{\operatorname{maj}(\sigma)}=\left[\begin{array}{c}
n \\
q_{1}, q_{2}-q_{1} \ldots, q_{t}-q_{t-1}, n-q_{t}
\end{array}\right]_{q} .
$$

It is shown in ([8], Proposition 1.3.17) that the inversion number has the same generating function over the same set:

$$
\sum_{\left\{\sigma \in S_{n} \mid \operatorname{Des}\left(\sigma^{-1}\right) \subseteq Q\right\}} q^{i n v(\sigma)}=\left[\begin{array}{c}
n \\
q_{1}, q_{2}-q_{1} \ldots, q_{t}-q_{t-1}, n-q_{t}
\end{array}\right]_{q} .
$$

The equidistribution of inversion number and major index over the set $\left\{\sigma \in S_{n} \mid\right.$ $\left.\operatorname{Des}\left(\sigma^{-1}\right) \subseteq Q\right\}$ follows immediately from these two equations, and their equidistribution over $S_{Q}$ itself follows from them as well by applying the inclusion-exclusion principle.

We conclude this paper by giving a direct bijective proof of these equidistribution results. The proof addresses only the case of $|Q|=1$; specifically, we assume $Q=\{b\}$, with $n=a+b$, and (preserving the notation of the last section) $\theta=(1, \ldots, b), \pi=(b+1, \ldots, n)$. (For the general case of $Q=\left\{q_{1}, \ldots, q_{t}\right\}$, the bijection is obtained by simply repeating the procedure described here $t$ times, where in the $i$-th round we assume $\theta$ to be any shuffle of $\mathbf{q}_{\mathbf{0}}, \ldots, \mathbf{q}_{\mathbf{i}-\mathbf{1}}$ and $\pi=\mathbf{q}_{\mathbf{i}}$ ). Thus equations (12) and (13) become:

$$
\sum_{\left\{\sigma \in S_{n} \mid \operatorname{Des}\left(\sigma^{-1}\right) \subseteq Q\right\}} q^{\operatorname{maj}(\sigma)}=\sum_{\left\{\sigma \in S_{n} \mid \operatorname{Des}\left(\sigma^{-1}\right) \subseteq Q\right\}} q^{i n v(\sigma)}=\left[\begin{array}{c}
n \\
a
\end{array}\right]_{q} .
$$

Our approach will proceed as follows. We will prove the generating function for inversion number by producing a bijection $\Psi: \mathcal{S}(\theta, \pi) \rightarrow \mathcal{P}(b, a)$ such that for $\tau \in \mathcal{S}(\theta, \pi)$, inv $(\tau)=$ $|\Psi(\sigma)|$. Then, utilizing the results of the last section, it will follow that $\Omega:=\Phi^{-1} \circ \Psi$ : $S_{Q} \rightarrow S_{Q}$ is a bijection from $S_{Q}$ to itself which maps inversion number to major index, proving the equidistribution of these statistics over $S_{Q}$.

The bijection $\Psi$ is very simple. Any shuffle $\tau$ of $\theta$ and $\pi$ is uniquely determined by the the weakly decreasing sequence $\left(t_{1}, \ldots, t_{a}\right)$ where $t_{i}$ is the number of elements of $\theta$ to the right of $b+i$ in $\tau$. Clearly, $0 \leqslant t_{i} \leqslant b$ for all $i$, and conversely any sequence $\left(t_{1}, \ldots, t_{a}\right)$ which is weakly decreasing with $0 \leqslant t_{i} \leqslant b$ for all $i$ uniquely determines a shuffle $\tau$ of $\theta$ and $\pi$. In this correspondence it is clear that $i n v(\tau)=\sum_{i=1}^{a} t_{i}$. Define $\Psi(\tau)=\operatorname{set}\left(t_{1}, \ldots, t_{a}\right)$.

To illustrate the bijection $\Omega: S_{Q} \rightarrow S_{Q}$ mapping inversion number to major index, 
consider the example of $n=7, b=4, \theta=(1,2,3,4), \pi=(5,6,7)$, and $\tau=5126374$. We have $\lambda:=\Psi(\tau)=\{4,2,1\}$. We calculate $\sigma:=\Phi^{-1}(\lambda)$ using the method described at the end of the last section (which is especially simple here because $d_{i}(\pi)=0$ for all $i$ since $\pi$ is increasing). As earlier, set $\sigma^{4}=\theta$, and the $i$-th insertion yields $\sigma^{4-i}$. At each stage we italicize the element of the major increment sequence which is furthest to the right among the "unused" elements of $\lambda$; this determines both $m_{i}$ and $k_{i}$ at that stage.

$$
\begin{array}{lll}
\operatorname{MIS}\left(\sigma^{4}, 7\right)=(1,2,3,4,0) & m_{3}=4, k_{3}=3 & \sigma^{3}=12374 \\
\operatorname{MIS}\left(\sigma^{3}, 6\right)=(2,3,4,1,5,0) & m_{3}=1, k_{3}=3 & \sigma^{2}=123674 \\
\operatorname{MIS}\left(\sigma^{2}, 5\right)=(2,3,4,1,5,6,0) & m_{3}=2, k_{3}=0 & \sigma^{1}=5123674 .
\end{array}
$$

Thus we have $\Omega(5126374)=5123674$.

As a final remark, we note that $\Omega$ is not only a bijection on the set $\left\{\sigma \in S_{n} \mid \operatorname{Des}\left(\sigma^{-1}\right) \subseteq Q\right\}$ but also on each individual inverse descent class contained in that set. This is true because the shuffle of $\theta$ and $\pi$ which is not well-mixed (i.e., the shuffle $\sigma^{0}$ in which $\pi$ is appended to the right of $\theta)$ is mapped to itself by $\Omega$ : $\Psi\left(\sigma^{0}\right)=(0,0, \ldots, 0)=\Phi^{-1}\left(\sigma^{0}\right)$. When $\Omega$ is iterated multiple times for the case of $|Q|>1$, this fact remains true at each stage, and hence, for all $i \in[n-1], i+1$ appears to the left of $i$ in $\Omega(\sigma)$ iff it does so in $\sigma$. Thus the descents of $(\Omega(\sigma))^{-1}$ are the same as those of $\sigma^{-1}$, i.e., $\sigma$ and $\Omega(\sigma)$ are in the same inverse descent class.

\section{References}

[1] D. Bressoud, Proofs and Confirmations: The Story of the Alternating Sign Matrix Conjecture, Cambridge University Press, 1999.

[2] D. Foata, On the Netto Inversion Number of a Sequence, Proceedings of the American Mathematical Society 19, 236-240 (1968).

[3] D. Foata and M. Schutzenberger, Major Index and Inversion Number of Permutations, Math. Nachr. 83, 143-159 (1978)

[4] A.M. Garsia and I. Gessel, Permutation Statistics and Partitions, Advances in Mathematics 31, 288-305 (1979)

[5] Hansraj Gupta, A New Look at the Permutations of the First n Natural Numbers, Indian Journal of Pure and Applied Mathematics 9:6, 600-631 (1978)

[6] J. Haglund, N. Loehr, and J. B. Remmel Statistics on Wreath Products, Perfect Matchings, and Signed Words, European Journal of Combinatorics 26, 195-232 (2005)

[7] P.A. MacMahon, Two Applications of General Theorems in Combinatory Analysis, Proceedings of the London Mathematical Society 15, 314-321 (1916).

[8] Richard Stanley, Enumerative Combinatorics, Volume 1.

[9] Richard Stanley, Ordered Structures and Partitions, Memoirs of the American Mathematical Society 119, 1-104 (1972) 